\title{
Disparités dans l'enseignement primaire et innovation pédagogique au Burkina Faso
}

Disparities in primary teaching and pedagogical innovation in Burkina Faso

Disparidades en la enseñanza primaria e innovación pedagógica en Burkina Faso

\section{Afsata Paré/Kaboré}

\section{CpenEdition}

\section{Journals}

Édition électronique

URL : http://journals.openedition.org/ries/2274

DOI : $10.4000 /$ ries.2274

ISSN : 2261-4265

Éditeur

Centre international d'études pédagogiques

Édition imprimée

Date de publication : 1 avril 2012

Pagination : 71-82

ISBN : 978-2-85420-594-7

ISSN : 1254-4590

\section{Référence électronique}

Afsata Paré/Kaboré, « Disparités dans l'enseignement primaire et innovation pédagogique au Burkina Faso », Revue internationale d'éducation de Sèvres [En ligne], 59 | avril 2012, mis en ligne le 06 février 2015, consulté le 19 avril 2019. URL : http://journals.openedition.org/ries/2274 ; DOI : 10.4000/ ries. 2274 


\section{Disparités dans l'enseignement primaire et innovation pédagogique au Burkina Faso}

\section{Afsata Paré/Kaboré}

Le Burkina Faso est un pays enclavé de l'Afrique de l'Ouest, aux ressources limitées. Convaincus de l'importance de l'éducation pour le développement socio-économique des pays, les décideurs, avec le soutien des partenaires et des institutions internationales, développent des stratégies afin d'être au rendez-vous des Objectifs du millénaire pour le développement ${ }^{1}$ de 2015 en ce qui concerne ce secteur. Cependant, il convient d'être réaliste et d'envisager pour un peu plus tard l'atteinte des objectifs de scolarisation universelle, particulièrement dans les zones rurales plus affectées par la pauvreté et les entraves socioculturelles à l'ouverture scolaire.

En effet, le contexte éducatif du Burkina Faso est marqué par des indicateurs d'accès et de qualité qui ne sont guère satisfaisants malgré les efforts consentis, surtout en milieu rural.

\section{LA SITUATION ÉDUCATIVE GLOBALE DU BURKINA FASO}

Le système éducatif au Burkina Faso comprend le sous-système formel et le sous-système non formel. Comme partout ailleurs, on dénombre au plan formel plusieurs paliers allant du préscolaire au supérieur. Pour ne pas entrer dans les détails, retenons qu'au terme de la loi d'orientation du 31 juillet $2007^{2}$, le préscolaire, l'enseignement primaire, le premier cycle de l'enseignement secondaire et l'éducation non formelle constituent ce qui est appelé « éducation de base » au Burkina Faso.

\section{Le sous-système formel \\ Au plan quantitatif et du rendement interne}

Au plan de l'accès, l'éducation préscolaire, quasi-exclusivement de l'ordre du privé, demeure un luxe accessible à une portion fort limitée des populations des grands centres urbains. En 2009-2010, le taux de préscolarisation atteignait 2,70\% au plan national ${ }^{3}$. La disparité existe entre régions, mais pas entre filles et garçons.

\footnotetext{
1. La Déclaration du millénaire de l'Organisation des Nations Unies a été signée le 8 septembre 2000 à New-York (NdIR). 2. $\mathrm{N}^{\circ} 013-2007 / \mathrm{AN}$.

3. Statistiques du ministère de l'Action sociale et de la solidarité nationale, qui a en charge l'éducation préscolaire.
} 
L'enseignement primaire, objet principal de notre propos, connaît également des disparités entre régions, disparités régionales doublées d'une disparité entre genres. Les statistiques scolaires du ministère de l'Enseignement de base et de l'Alphabétisation $\left(\mathrm{MEBA}^{4}, 2011\right)$ présentent un taux brut de scolarisation (TBS) au primaire qui, au plan national, était en 2010-2011 de $77,6 \%$, soit $80,2 \%$ pour les garçons et $75 \%$ pour les filles. La croissance des effectifs est continue, avec un taux de croissance moyen de $9,03 \%$ pour la période 2000-2007.

Dans l'enseignement secondaire, le taux brut de scolarisation est très faible, soit $22,2 \%$ en 2009-2010, avec également de fortes disparités entre régions et entre genres ( $25,7 \%$ pour les garçons et $18,7 \%$ pour les filles). Pourtant, le rythme d'accroissement des effectifs scolaires du secondaire a été en progression constante pour atteindre $12,6 \%$ à partir de $2007-2008^{5}$.

Le taux de croissance de la population reste élevé $(2,68 \%$ selon les chiffres de 2006, soit 16241000 habitants en 2010), ce qui rend la charge éducative fort importante et explique une progression plutôt lente des taux de scolarisation.

Les effectifs de l'enseignement supérieur connaissent une croissance soutenue, avec un taux d'accroissement moyen de 7,47 \% par an entre 2004-2005 et 2009-2010. Toutefois, le nombre d'étudiants pour 100000 habitants est très faible (298 en 2009-2010). Là encore, ce sont les zones rurales qui sont le plus défavorisées, étant donné la concentration des établissements d'enseignement secondaire et supérieur dans les villes.

Au plan du rendement, on note de forts taux de redoublements et de déperdition et donc de faibles taux de survie. Cela signifie un gaspillage des ressources qui, dans un pays comme le Burkina Faso, sont déjà rares. Le taux de survie s'améliore cependant au fur et à mesure que l'on évolue vers les niveaux scolaires les plus élevés.

En ce qui concerne le primaire, le rendement interne, bien qu'en amélioration, reste bas : taux d'achèvement de $46 \%$ et de succès au certificat d'études primaires (CEP) de $66 \%$ en 2009-2010; taux de redoublement en nette régression, étant donné la politique vigoureuse adoptée sur ce plan qui interdit les redoublements à l'intérieur du même cycle ${ }^{6}$.

Au secondaire, en 2009-2010, les taux de redoublements sont de $25 \%$ au premier cycle et de $23 \%$ au second cycle, avec de faibles performances moyennes dans les principales disciplines, toutes inférieures à la moyenne à l'exception du français.

4. Ce ministère s'intitule désormais « ministère de l’Éducation nationale et de l'Alphabétisation » (MENA).

5. Statistiques scolaires et universitaires, 2011, DEP/MESSRS (Direction des études et de la planification du ministère des Enseignements secondaire, supérieur et de la Recherche scientifique qui est à présent simplement le ministère des Enseignements secondaire et supérieur : MESS).

6. Les six années de l'école primaire classique sont découpées en sous-cycles : cours primaire $1^{\text {re }}$ et $2^{\mathrm{e}}$ années $\left(\mathrm{CP}_{1}\right.$ et $\left.\mathrm{CP}_{2}\right)$; cours élémentaire $1^{\mathrm{re}}$ et $2^{\mathrm{e}}$ années (CE1 et $\left.\mathrm{CE}_{2}\right)$ et cours moyens $1^{\mathrm{re}}$ et $2^{\mathrm{e}}$ années $\left(\mathrm{CM}_{1}\right.$ et $\left.\mathrm{CM}_{2}\right)$. 
Dans l'enseignement supérieur, les indicateurs de survie en 2008 (DEP/ MESSRS) sont de $32 \%$ en quatrième année d'université (année de maîtrise). Cependant, beaucoup d'étudiants se contentent d'intégrer le marché de l'emploi à $\mathrm{bac}+2 \mathrm{ou} \mathrm{bac}+3$.

Sans s'attarder sur l'historique des différents indicateurs, il convient de préciser qu'au fil des ans, les indicateurs du système éducatif ont généralement connu une amélioration continue : qu'il s'agisse des taux d'accès, des taux bruts de scolarisation, des taux d'achèvement ou des disparités entre les régions et les sexes. On souhaiterait que cette évolution soit plus rapide qu'elle ne l'est actuellement. Des mesures plus vigoureuses sont mises en œuvre pour 48 communes identifiées comme prioritaires, du fait de leur retard par rapport aux autres. L'impact positif de ces mesures devrait permettre d'améliorer plus rapidement les différents indicateurs à l'échelle nationale. Malgré tout, il est à craindre que le Burkina Faso ne soit pas au rendez-vous de l'objectif de scolarisation universelle de 2015.

\section{Le rendement externe}

Au plan socioculturel, on note un impact positif important de la fréquentation scolaire. Le Rapport d'État du système éducatif national (Resen) montrait en effet en 2009 que chez les Burkinabés, le niveau d'adoption des comportements favorables à la santé, au bien-être de la famille, à l'éducation des enfants, croissait avec l'élévation de leur niveau scolaire. Au plan économique, le Resen mettait également en évidence la corrélation entre les niveaux de revenus des employés et leur niveau d'éducation, quel que soit le domaine d'exercice, que celui-ci soit du secteur informel ou formel.

À côté de ces motifs de satisfaction, il y a lieu de relever également plusieurs motifs d'insatisfaction. En effet, on note un important taux de chômage des jeunes diplômés de 25-34 ans (10,5 \% en 2007), particulièrement de ceux du supérieur pour lesquels ce taux atteignait $21,3 \%$ en 2007. L'enseignement supérieur semble donc produire deux fois plus de diplômés que le nombre d'emplois de cadres supérieurs et moyens (900 environ par an) disponibles. Le problème des filières de formation se pose également, dans la mesure où la majorité des sortants sont issus de filières dont les opportunités d'emploi sont relativement faibles (lettres et sciences humaines).

L'enseignement primaire, à son niveau actuel de qualité, est insuffisant pour garantir une alphabétisation définitive. Le Resen montre que la probabilité d'être alphabétisé après six années de scolarité (correspondant à la durée de l'école primaire au Burkina Faso), est de 45,3\%, alors que la moyenne africaine s'élève à $68 \%{ }^{7}$. La performance de l'école primaire burkinabè ${ }^{8}$ se situe donc très en dessous de la moyenne africaine (23 points d'écart). Ce rapport Resen précise

7. Unesco-Breda 2007, cité par le Resen.

8. Le terme « burkinabè » est invariable en langue fulfuldé ( $N d I R)$. 
que dans son état actuel, il faudrait environ dix années de scolarité au Burkina Faso (correspondant à la fin du premier cycle de l'enseignement secondaire), pour que la probabilité d'alphabétisation soit de $100 \%$.

Enfin, notons que les déscolarisés précoces (avant la fin du primaire) ou moins précoces (entre la fin du primaire et les débuts du secondaire), qu'ils soient issus ou non des zones rurales, n'ont pas acquis par l'école des compétences particulières de travail mais se détournent quand même des emplois de leurs parents, à savoir les emplois du secteur primaire (agriculture, élevage). Cette situation ne fait qu'accroître la proportion de jeunes et d'adultes improductifs et freine l'élan de développement du pays.

Des formules alternatives de scolarisation ont vu le jour depuis quelques années, qui tentent de rendre l'éducation mieux adaptée aux réalités socioculturelles et aux besoins du pays. Au plan du sous-système formel, on peut retenir l'éducation bilingue (EB) par exemple. Celle-ci assure un enseignement aussi bien en français qu'en langue locale, intègre les valeurs culturelles locales ainsi que la production (apprentissages manuels) dans les programmes. Les différentes études montrent qu'il s'agit d'une alternative prometteuse en termes de rendement. Nous y reviendrons.

\section{Le sous-système non formel d'éducation}

Le sous-système non formel, tout en s'adressant à ceux qui n'ont pas eu accès à l'école ou qui ont été précocement déscolarisés, constitue aussi une alternative éducative qui tente de pallier les insuffisances de l'éducation formelle.

La majorité de la population burkinabè est analphabète. En 2005, on estimait à $76 \%$ la proportion de la population ne sachant ni lire ni écrire dans une langue quelconque. Toutefois, le taux d'alphabétisation est passé de 18,9\% en 1994 à 23,6 \% en 2005. C'est dire qu'au Burkina Faso, l'éducation non formelle occupe une place importante dans le système éducatif et dans l'éducation des populations. Dans ce sous-système, on trouve l'alphabétisation des adultes et les divers centres d'éducation de base pour jeunes. C'est un sous-système qui, dans la pratique, a la spécificité de s'appuyer principalement sur les langues nationales. Par ailleurs, c'est une forme d'éducation qui, outre les apprentissages théoriques, procède aussi à des apprentissages techniques et professionnels.

Les statistiques relatives à l'éducation non formelle sont en général assez incomplètes et peu disponibles. Les dernières dont nous disposons sont celles de 2006 et 2007. En 2006, le taux d'alphabétisation des adultes (les 15 ans et plus) était de 28,7\% par rapport à 23,2 \% l'année précédente (MEBA 2007). Des disparités existent entre les hommes $(36,7 \%)$ et les femmes (21\%). Les femmes constituent pourtant ces cinq dernières années, la plus grande partie des effectifs des centres d'alphabétisation. Le tableau qui suit donne un aperçu de l'alphabétisation initiale (AI) et de l'alphabétisation en formation complémentaire de base (FCB) en 2007-2008. On y voit que les effectifs féminins sont 
Tableau $\mathbf{n}^{\circ} 1$

Résultats en alphabétisation AI et FCB en 2007-2008

\begin{tabular}{|l|c|c|c|c|c|c|}
\hline & \multicolumn{3}{|c|}{ Alphabétisation initiale } & \multicolumn{3}{c|}{ Formation complémentaire de base } \\
\cline { 2 - 7 } & F & H & Écart & F & H & Écart \\
\hline \% des effectifs totaux & 61,96 & 38,04 & 23,92 & 60,55 & 39,45 & 21,10 \\
\hline Taux de rétention & 91,9 & 90,1 & 1,8 & 90,90 & 90,50 & 0,40 \\
\hline Taux d'admission & 83,00 & 82,7 & 0,3 & 82,00 & 84,70 & $-2,70$ \\
\hline
\end{tabular}

Source : conçu à partir des données du rapport du Plan décennal de développement de l'enseignement de base (PDDEB).

plus nombreux mais que leur taux d'admission aux examens semble moindre que celui des effectifs masculins en FCB. On peut néanmoins affirmer qu'il y a une nette amélioration par rapport aux années passées.

Outre que la présentation de l'éducation non formelle est indispensable pour se faire une idée globale du système éducatif, il y a un intérêt supplémentaire à l'évoquer, bien que notre propos concerne l'enseignement primaire qui relève du sous-système formel. En effet, l'éducation non formelle a inspiré l'éducation formelle dans une de ses innovations qu'est l'école bilingue. Dans la suite de notre propos, il sera question du sous-système formel d'éducation, particulièrement de l'enseignement primaire.

\section{DisPARITÉS ENTRE MILIEUX URBAIN ET RURAL DANS L'ENSEIGNEMENT PRIMAIRE}

Aussi bien au plan quantitatif que qualitatif, le milieu rural burkinabè connaît plus de difficultés que le milieu urbain. C'est là que les pesanteurs culturelles et les réalités socioéconomiques ne militent pas en faveur d'une approche positive de l'école par les enfants et les parents. Tout cela constitue des limites à l'atteinte d'une efficacité acceptable de l'école dans ces zones.

Les disparités entre les sexes sont plus manifestes dans les zones rurales. Par exemple, les effectifs des filles et des garçons sont presque équilibrés en milieu urbain (49,5\% de filles), cependant qu'en milieu rural, l'écart reste encore assez important (45,7\% de filles, soit près de cinq points).

Du point de vue de l'organisation et des conditions scolaires (enseignants, fournitures en eau ou électricité, distance à parcourir par les élèves pour aller à l'école, etc.), le milieu rural est en général moins bien loti.

Il y a moins de classes simples en milieu rural $(87,54 \%$ contre $94,60 \%$ en milieu urbain), de même qu'il y a moins de classes multigrades en milieu urbain $(0,72 \%$ contre $12,07 \%$ en milieu rural). Par contre, les classes à double flux (par demi-journée), sont plus nombreuses en milieu urbain (4,68 \%) qu'en milieu rural $(0,40 \%)$. La tendance est de rendre toutes les classes simples. 
Cependant, l'insuffisance d'infrastructures et/ou d'enseignants oblige à maintenir des classes multigrades ou à double flux.

On note aussi que les enseignants les moins expérimentés se retrouvent surtout en milieu rural $(7,17 \%$ ont une expérience de plus de quinze ans par rapport à 23,9\% en milieu urbain). Les plus qualifiés sont en proportion moindre en milieu rural, où l'on trouve surtout des instituteurs adjoints certifiés comparativement au milieu urbain, où exercent surtout des instituteurs certifiés ${ }^{9}$.

En outre, selon les statistiques 2010-2011 du ministère de l'Éducation nationale et de l'alphabétisation, les écoles urbaines sont dans l'ensemble mieux pourvues en eau potable ( $66 \%$ contre $41,7 \%$ en zone rurale), électricité $(43,8 \%$ contre $5,8 \%$ ), latrines fonctionnelles $(84,7 \%$ contre $62,1 \%)$, clôture $(35,4 \%$ contre 1,7\%). Mais les écoles rurales ont plus de cantines scolaires (80,5\% contre $65,1 \%$ en zone urbaine), de terrains de sport (78,3 contre $70 \%)$. La couverture en matière de cantines n'est pas encore totale en milieu rural, alors qu'elles constituent un moyen important de rétention scolaire.

$\mathrm{Au}$ plan du rendement interne, les taux de redoublement ne sont pas plus importants en zone rurale. Ils sont même moindres en 2010-2011, mais les évaluations révèlent des acquisitions plus importantes en français, en mathématiques et en sciences chez les enfants vivant en milieu urbain. On peut sans doute avancer ici les problèmes d'évaluation et de décision de redoublement évoqués par le Programme d'analyse des systèmes éducatifs (Pasec) ${ }^{10}$ de la Confemen ${ }^{11}$, qui souligne "de fortes incohérences dans les décisions de redoublement (...) qui ne se fondent pas sur une sélection objective des élèves sur la base de leurs acquis scolaires» (p. 43).

Il est possible en effet qu'en termes de standards de performances scolaires, les enseignants soient moins exigeants en milieu rural, ce qui expliquerait la proportion moins importante de redoublants dans ce milieu, avec cependant des acquis scolaires plus bas. Nous restons toutefois nuancée dans notre interprétation, dans la mesure où nous n'avons pas affaire à la même année de référence des données utilisées, même si la différence des taux d'achèvement en faveur du milieu urbain et des plus favorisés (la pauvreté sévit plus en milieu rural) en dit également long sur les difficultés plus importantes auxquelles fait face le milieu rural (voir le tableau 2).

Pour améliorer le rendement interne de l'école en général et en milieu rural en particulier, des actions sont menées qui touchent autant les parents (sensibilisation, promotion de leur l'alphabétisation), l'environnement scolaire (par exemple études surveillées en dehors des heures de classes) que l'approche même de l'école.

9. La catégorisation des enseignants par ordre décroissant de qualification est la suivante : instituteur principal (IP) ; instituteur certifié (IC) ; instituteur adjoint certifié (IAC) ; instituteur adjoint (IA).

10. Jean-Marc Bernard, Odile Simon, Katia Vianou (2005).

11. Confemen : Conférence des ministres de l'Éducation des pays ayant le français en partage. (NdLR). 
Tableau ${ }^{\circ} 2$

Résumé des différences de taux d'achèvement au primaire

\begin{tabular}{|c|c|}
\hline Milieu de vie de l'élève & Taux d'achèvement au primaire \\
\hline Urbain & $61,0 \%$ \\
\hline Rural & $28,0 \%$ \\
\hline
\end{tabular}

Source : RESEN (2008)

\section{L'ÉDUCATION BILINGUE, UNE STRATÉGIE POUR AMÉLIORER LE RENDEMENT DE L’ÉCOLE}

Dans la gamme des initiatives existantes en faveur de l'éducation en milieu rural, nous nous intéresserons en particulier à la promotion de l'éducation bilingue.

\section{Les origines}

L'éducation bilingue, telle qu'elle est pratiquée aujourd'hui, est une autre approche de l'école qui est née en zone rurale, mais dont celle-ci n'a plus l'apanage. Elle a eu ses prémisses à l'école primaire avec l'enseignement bi/plurilingue issu de la réforme éducative nationale expérimentée pour la première fois entre 1979 et 1984 et interrompue à la fin de l'année scolaire 1983-1984. «Une de ses innovations a été l'utilisation, comme médiums d'enseignement, de trois langues nationales : le fulfulde, le dioula et le mooré. C'était la première fois qu'il était question d'utiliser les langues nationales dans le système d'éducation formelle. L'innovation a commencé dans 28 écoles expérimentales essentiellement implantées en zone rurale. Les premières promotions d'élèves sont allées jusqu'en cinquième année (CM1). Toutefois, la réforme a été arrêtée en 1984, sans évaluation» (Nikiema et Pare Kabore 2010, p. 21).

Avant cette réforme interrompue, on était plutôt dans une situation de répartition des rôles entre l'éducation non formelle et l'éducation formelle, la première se faisant en langues nationales et la seconde en français. C'est dans ce contexte que l'éducation bilingue dont il est question ici a cependant vu le jour en 1994, "car malgré la situation évoquée ci-dessus, les États généraux de l'éducation (1994) ont réaffirmé l'opportunité d'utiliser les langues nationales dans le système d'éducation formelle. Un cadre légal pour le recours aux langues nationales a par ailleurs été fourni dans la loi d'orientation de l'éducation de $1996 »$ (Nikiema et Pare/Kabore, 2010, p. 22).

L'éducation bilingue a été mise en œuvre dans le cadre de la coopération du MEBA avec l'OSEO ${ }^{12}$. Ce concept d'école a évolué à partir du contexte rural, pour ensuite intéresser les milieux urbains. Il a débuté à la rentrée 1994. Au

12. OSEO : Euvre suisse d'entraide ouvrière, récemment rebaptisée Solidar Suisse ( $N d I R)$. 
départ, il s'agissait, à la demande d'une association, de mettre en œuvre une formule de "scolarisation bilingue accélérée » pour des enfants de deux villages, Nomgana et Goué, non loin d'Ouagadougou, la capitale. Cette association villageoise avait bénéficié de la "méthode ALFAA $^{13}$ » d'alphabétisation, dont le succès auprès des adultes membres de l'association avait entraîné la demande d'en faire bénéficier les enfants non scolarisés de neuf ans ou plus (qui avaient donc passé l'âge d'être recrutés à l'école primaire, tout en étant trop jeunes pour fréquenter les centres d'alphabétisation des adultes). "Afin de combler le retard accusé par ces enfants, et en tenant compte du fait qu'ils étaient psychologiquement plus mûrs, l'option a été faite d'essayer une scolarisation de quatre ans au lieu des six ans standards. 55 enfants ont été recrutés et alphabétisés en mooré par l'association selon la méthode de l'alphabétisation formation intensive (AFI) alors en cours ; les maîtres ont été sélectionnés et formés de manière intensive pendant les vacances scolaires. Les supports didactiques ont été élaborés et l'expérimentation enclenchée en utilisant le mooré, la langue du milieu, pendant les deux premières années pour les premiers apprentissages, cependant qu'ils apprenaient le français selon la méthode ALFAA» (Nikiema et Pare Kabore, 2010, p. 25).

\section{L'évolution de l'approche et ses résultats}

Depuis la création des premières écoles, la formule de l'éducation bilingue a évolué et elle est aujourd'hui un continuum allant du préscolaire ("espaces d'éveil éducatif ou $3 \mathrm{E}$ ») aux «collèges multilingues spécifiques », en passant par les écoles primaires bilingues (EPB).

L'éducation primaire bilingue (EPB) concerne les enfants âgés de 7 à 12 ans et a pour principaux objectifs :

- d'associer l'acte d'apprendre à l'acte de produire ;

- de réconcilier l'école avec le milieu, en y intégrant les valeurs culturelles positives du milieu et en impliquant la communauté environnante ;

- d'utiliser et valoriser les connaissances de l'enfant dans une langue nationale, de valoriser les acquis de l'alphabétisation dans cette langue.

Couvrant au départ l'ensemble du territoire et d'une langue nationale (le moore), l'EPB concerne aujourd'hui huit langues nationales, chacune étant équipée et documentée (manuels d'élèves, guides du maître) pour les différentes disciplines et les différentes classes. Ces langues sont : le bissa, le dagara, le dioula, le fulfulde, le gulmancéma, le lyélé, le mooré, le nuni. D’autres sont en passe d'être ajoutées. C'est dire l'important travail de recherche effectué par les linguistes pour arriver à un tel stade. Les écoles primaires bilingues ont un cycle de cinq ans (au lieu de six dans le système classique) et continuent à se multiplier, tant en milieu rural qu'en milieu urbain.

13. Il s'agit d'une méthode d'apprentissage du français à partir des acquis de l'alphabétisation en langues nationales. 
Tableau $\mathbf{n}^{\circ} 3$

Résultats d'écoles classiques et d'écoles bilingues en français et en mathématiques

\begin{tabular}{|l|l|c|c|c|}
\hline \multirow{4}{*}{ Année } & \multicolumn{1}{|c|}{ Type d'école } & Niveau & Français & Mathématiques \\
\hline \multirow{4}{*}{2005} & Classique & $1^{\text {re }}$ année & 42,1 & 36,0 \\
\cline { 2 - 5 } & Bilingue & $1^{\text {re }}$ année & 43,0 & 45,4 \\
\cline { 2 - 5 } & Classique & $4^{\mathrm{e}}$ année & 43,1 & 38,0 \\
\cline { 2 - 5 } & Bilingue & $4^{\mathrm{e}}$ année & 45,2 & 45,8 \\
\hline \multirow{3}{*}{2008} & Classique & $1^{\text {re }}$ année & 42,3 & 42,9 \\
\cline { 2 - 5 } & Bilingue & $1^{\text {re }}$ année & 43,7 & 54,9 \\
\cline { 2 - 5 } & Classique & $4^{\mathrm{e}}$ année & 45,4 & 39,4 \\
\cline { 2 - 5 } & Bilingue & $4^{\mathrm{e}}$ année & 38,1 & 38,0 \\
\hline
\end{tabular}

Source : DEP MEBA (2009), Rapport sur l'évaluation des acquis scolaires 2007-2008.

La production fait partie du programme de ces écoles et le choix du type de production se fait localement (jardinage, menuiserie, élevage, etc., selon les cas). Par ailleurs, les valeurs culturelles locales y sont intégrées et animées par les parents (contes, devinettes, proverbes, musique, danses, etc.), selon les possibilités.

L'utilisation de la langue nationale et du français est organisée de manière à aboutir à une bonne maîtrise aussi bien orale qu'écrite de chacune d'elles. Dans la logique d'un bilinguisme additif ${ }^{14}$, leur progression respective est inversement proportionnelle dans le temps. On passe de $90 \%$ pour la langue nationale et $10 \%$ pour le français en $1^{\text {re }}$ année à $10 \%$ pour la première contre $90 \%$ pour la seconde en $5^{\mathrm{e}}$ année. Cette inversion complète du pourcentage de temps accordé à la langue nationale et au français en fin de cycle se justifie par ailleurs par le fait que les élèves de ces écoles sont soumis à l'examen du certificat d'études primaires (CEP) de l'enseignement classique entièrement en français. C'est dire qu'il n'y a pas encore de certification propre à l'éducation bilingue. Malgré cela, les acquis en mathématiques et en français (voir tableau 3) tout comme les résultats au CEP des élèves des écoles primaires bilingues n'ont rien à envier à ceux des élèves de l'école classique et sont d'ailleurs généralement meilleurs. Il en va de même pour les résultats au CEP, où les écoles bilingues ont enregistré 73,97 \% (2007) et 61,43\% (2008) de succès contre $66,83 \%$ et 58,34\% pour les écoles classiques. On peut dès lors comprendre l'extension quantitative de ces écoles, qui sont passées de 2 en 1994 à 117 en 2006-2007, dont 80 publiques et 37 privées. En 2006-2007, l'effectif d'élèves inscrits s'y élevait à 15478 . En 2010-2011, ils étaient 21 170, soit 5479 élèves en milieu urbain (2680 garçons et 2799 filles) et 15691 en milieu rural (8122 garçons et 7569 filles). Certes,

14. Système dans lequel on vise le maintien des deux langues tout au long du processus éducatif. 
cela représente un peu moins d'1\% de l'effectif total des élèves de l'enseignement primaire mais l'État s'est engagé pour une extension significative de l'éducation bilingue, étant donné ses résultats intéressants et son coût financier moins élevé que l'école classique (Remain Kinda 2003). Une telle perspective suppose néanmoins une attention particulière portée aux facteurs qui pourraient jouer négativement sur la qualité.

Le processus de communalisation vers lequel évolue aujourd'hui le Burkina Faso, associé à la responsabilisation des communes en matière d'éducation de base, semble augurer d'une opportunité d'adaptation accrue de l'école au milieu, surtout lorsqu'il s'agit des écoles primaires bilingues, notamment dans leurs dimensions production et culture. En effet, des possibilités s'offrent de pouvoir tenir compte des réalités locales pour mettre en œuvre ces dimensions. Il faudra cependant prendre les précautions nécessaires afin que cela ne se fasse pas au détriment de la création de valeurs nationales, pour une identité nationale burkinabè.

$\mathrm{Au}$ demeurant, il y a fort à parier que, dans la pratique, le milieu rural et le milieu urbain se distinguent dans la manière dont les dimensions production et culture de l'éducation bilingue sont organisées. Bien que la question n'ait pas fait l'objet d'une observation de notre part, on peut imaginer que le milieu rural bénéficie de plus d'atouts (davantage de parents pétris des valeurs culturelles, des parents plus disponibles pour la formation des élèves sur ces questions, des espaces pour l'exploration et la pratique de travaux manuels, etc.). Il peut être intéressant de se pencher de plus près sur cette comparaison afin d'identifier les facteurs favorables ou défavorables à l'insertion des écoles bilingues dans les différents milieux et de faire des propositions pouvant garantir une meilleure insertion.

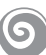

Différentes études au sujet de l'éducation bilingue (Alidou et al., 2008 ; Nikiema et al., 2010) montrent la qualité des acquisitions instrumentales qu'elle permet aux apprenants. En outre, l'éducation bilingue œuvre aussi à la promotion de la culture et des langues nationales et donc à la préservation et à l'enrichissement du patrimoine traditionnel. Cela constitue déjà un aspect en lien avec le rendement externe de l'école, celui-ci étant renforcé par les compétences en production que les enfants développent.

Cependant, environ $1 \%$ seulement des élèves burkinabè fréquente les écoles primaires bilingues. Aussi, dans la perspective d'une extension significative de cette éducation, les études insistent sur la nécessité de prendre les dispositions nécessaires afin que la qualité qui lui est reconnue soit préservée. Entre autres, il importe que la formation initiale et continue des enseignants demeure une préoccupation de tous les instants, que les outils didactiques soient disponibles en quantité et en qualité, que le suivi du processus par les conseillers 
pédagogiques soit réellement fait, que la recherche continue à s'intéresser à la question pour être dans une dynamique d'amélioration constante, que l'on adopte une politique vigoureuse pour enrichir l'environnement lettré en langues nationales, etc. En ce sens, les autorités communales devraient être accompagnées par les spécialistes et les conseillers pédagogiques pour une prise en charge efficace de l'éducation dans leur commune.

Au plan national, cet effort d'enrichissement de l'environnement lettré en langues nationales serait en continuité avec la volonté affichée par les décideurs de valoriser notre patrimoine culturel à travers les grandes manifestations comme la Semaine nationale de la culture (SNC), le Salon international de l'artisanat d'Ouagadougou (SIAO), le Festival panafricain du cinéma de Ouagadougou (FESPACO). Ces manifestations sont des cadres à travers lesquels nos valeurs culturelles, au sens large du terme, sont diffusées, connues et reconnues. En milieu rural singulièrement, l'éducation bilingue devrait permettre de rapprocher les enfants et les parents, car elle leur offre l'occasion de partager des connaissances à l'écrit en langues nationales, de renforcer mutuellement leurs compétences en la matière et dans les activités de production. En définitive, on tendrait ainsi à surmonter la situation de diglossie dans laquelle nous sommes actuellement.

Toutefois, il serait illusoire de penser que l'éducation bilingue comporte en elle-même toutes les solutions aux problèmes du système éducatif du Burkina Faso. La croissance lente du taux de scolarisation demeure un problème à résoudre en lien avec les politiques en matière de lutte contre les discriminations. Le problème de l'école dans les zones nomades du nord du pays reste posé car les mesures adoptées (sensibilisation, cantines scolaires, écoles du berger ${ }^{15}$, etc.), malgré certains résultats encourageants, n'ont pas encore eu raison de toutes les pesanteurs existantes. La discrimination éducative des filles, bien qu'en recul, reste un problème à régler, notamment en milieu rural. Des initiatives dans ce sens sont promues et elles pourraient faire l'objet d'une observation systématique et soutenue, afin d'en tirer le meilleur pour enrichir le système éducatif dans son ensemble.

\section{BiBLIOGRAPHIE}

BERNARD J. M., SIMON O., VIANOU K. (2005) : Le redoublement: mirage de l'école africaine? CONFEMEN, Dakar.

BURKINA FASO (2007) : Loi d'orientation de l'éducation au Burkina Faso. Ouagadougou : Premier Ministère/MESSRS/MEBA.

DEP/MEBA (2011) : Annuaires statistiques. Ouagadougou, DEP/MEBA.

15. « L'école du berger » est une formule alternative d'éducation de base non formelle, conçue par l'Association Andal \& Pinal (littéralement «Savoir et Éveil », en fulfuldé) et qui tient compte des préoccupations, des besoins et de la demande des éleveurs. Une école pour ainsi dire «à la carte» (Journal Observateur Paalga, mai 2007). 
DEP MEBA (2009): Rapport sur l'évaluation des acquis scolaires 2007-2008. Ouagadougou.

DEP/MESSRS (2010) : Annuaires statistiques. Ouagadougou, DEP/MESSRS.

MESSRS/MEBA (2009) : Rapport d'état du système éducatif national (RESEN). Burkina Faso.

Ministère de l'Action sociale et de la Solidarité nationale (2010): Statistiques $d u$ préscolaire, Ouagadougou.

NIKIEMA N. et PARE/KABORE A. (2010) : Rapport d'étude LASCOLAF. OIF.

OCECOC/MESSRS (2008) : Rapport d'évaluation des acquis scolaires dans l'enseignement secondaire. Burkina Faso.

RÉMAIN-KINDA E. C. (2003) : L'impact d'une généralisation de l'éducation bilingue sur le Plan de Développement Décennal de l'Éducation de Base: cas des écoles bilingues. Mémoire de fin de formation IEPD de l'ENSK, Koudougou, Burkina Faso. 